\title{
APPLYING AN ENTROPIC ANALYSIS TO LOCATE RAPID TRANSIT LINES IN SPRAWLED CITIES
}

\author{
FRANCISCO A. ORTEGA, RAMÓN PIEDRA-DE-LA-CUADRA \& SOLY VENTURA \\ Higher Technical School of Architecture, Universidad de Sevilla, Spain.
}

\begin{abstract}
Urban sprawl is a phenomenon that leads to an extensive use of motorized transport modes with negative environmental impacts such as congestion, time wasted in traffic jams, air and noise pollution and additional costs incurred by using non-renewable energy. Increasing the existing infrastructures is a decision, which often generates the installation of new urban settlements, whose degree of isolation is mitigated with a new increase in the demand for transport. This vicious circle can be broken by reducing the need of transport imposed by the urban model, which is only possible by bringing citizens closer to those services they demand. In the model of sprawled city, housing predominates as land use in the residential areas, where other complementary uses (such as commercial, cultural, institutional and industrial ones) are excluded in the urban development. When the urban districts don't present enough complexity, an increase in traffic density between different zones into the city arises. Such forced mobility could be reduced if the functional diversity of the districts were greater, or if there was an urban rapid transit system connecting the areas that generate the greatest imbalances. To measure the complexity of the urban districts system, the Information Theory developed in the 1960s proposes the use of urban entropy. The paper addresses the problem of locating a rapid transit line (metro, tram, BRT) with the objective of maximize the functional diversity of the districts traversed by the alignment. In order to illustrate the proposed model a computational experience is carried out by using data from the metropolitan area of Seville (Spain).

Keywords: entropic analysis, rapid transit line, urban diversity.
\end{abstract}

\section{INTRODUCTION}

The increase in transport demand caused by the urban model of sprawled city generates a massive use of the means of locomotion that saturate the roads destined to mobility and produce periodic episodes of pollution [1].

In general, urban sprawl refers to certain forms of city spatial expansion toward suburbs and peripheral areas with, low density, single-use, extensive road and highway networks, cardependent, open up vast space of territory, scattered and ribbon development in an mono-centric urban structure [2].

From a socio-economic perspective, urban sprawl leads to excessive infrastructure and public service costs, the decline of public space, reducing social cohesion, loss of a sense of community, loss of cultural values, increase of the income inequality and polarization, traffic congestion, longer travel distances and limited access, especially for non-driver people [3].

The urban mobility analysis reveals the existing congestion patterns. Daily trips can be either 'mandatory' (workplace-home) or 'voluntary' (shopping, leisure, visits). The former is often performed within fixed schedules while the latter complies with variable and discretionary schedules. Mandatory trips are mainly responsible for the peaks in circulation flows, implying that about half the congestion in urban areas is recurring at specific times of the day and on specific segments of the transport system. Urban transportation is a highly dynamic system where one component impacts on others with retroactive (feedback) effects are also to be expected. Congestion is a classic example of feedback loop. Pressures on the owners of transport infrastructure (usually the public sector) by different user groups being impacted by congestion may often result in the addition of new capacity such as new or wider roads. 
A capacity expansion often results in lower friction to mobility and this may impact on urban sprawl, since people may travel longer distances for a similar amount of time. The outcome is likely to become an increase in trip lengths, more trips and eventually more congestion. Consequently, users, through their choices of transportation modes, are recursively influencing into the development of the urban transport system. Several North American cities found themselves in a vicious cycle that triggered an increasing reliance on road transportation and on the automobile. This vicious circle can be mitigated if urban population and economic growth remain stabilized or if alternatives, such as public transit systems, are more readily available [4].

In the sprawl city model, districts only serve for a residential function and they are emptied of other contents. The complexity of the urban setting is based on the diversity of functions that are inside located (residence, education, health, commerce, industry, etc.). Numerous researchers ensure that the shortage of urban complexity, that the districts of a city exhibit, causes an increase in traffic density [5]. Assuming this hypothesis, promoting the functional diversity of the districts should consistently imply a reduction in the forced mobility of their inhabitants.

On the opposite side, the concept of compact city has been widely adopted as a mechanism for controlling and regulating urban sprawl by promoting a more intensive use of urban land, greater dwelling density, greater mixing of land uses and higher degrees of accessibility, providing opportunities for using public transport, walking and cycling. Burton [6] summarizes the positive effects of urban compactness pointing out that a higher degree in that characteristic improves public transport use, reduces social segregation and provides better access to facilities.

However, urban intensification is, by self, unlikely to lead to the achievement of better environmental and social outcomes. As was pointed out by the Commission of the European Communities (CEC), the transport plays a key role in efforts to reduce regional and social disparities and achieve territorial cohesion (CEC [7]; CEC [8]). In Ref. [8], the CEC concludes that land-use policies to increase urban density or mix land use, without accompanying transport measures to restrict car use and provide alternatives to car use, will have only limited effect. Unless there are supporting transport policies, urban intensification may simply attract more people and cars towards already congested areas. This assumption has provided a justification for financing infrastructure investments in peripheral regions, in order to compensate these regions for their lack of accessibility.

Maximizing coverage of trip demand is a common objective when locating transit lines because it is possible, by means of such infrastructures, to improve the population's mobility. However, some authors argue that certain investments may lead to increasing rather than reducing regional disparities $[9,10]$. Under this perspective, Ref. [11] explore the effects of large-scale transport infrastructure investments (carried out in surface transport modes during period 1992-2004 in Spain) on the assessment of regional cohesion. Reference [12] develop a multi-criteria assessment methodology where are included, for a regional scale, linking network analysis, spatial geography, regional economic and environmental issues. They conclude that, in the first stages of a network development, transport policy is usually efficiency-oriented, but as infrastructure provision increases, the optimal strategy for implementing transport infrastructures must also address to the acquiring of a trade-off between criteria of efficiency, cohesion, and environment.

To face with urban sprawl and growing traffic congestion, several cities throughout the world have turned to metro, rapid rail transit (RRT) and light rail transit (LRT) systems. Building rapid transit systems requires long term commitments and sizeable capital 
investments. What constitutes a good infrastructure configuration is by no means obvious, due to planners, engineers, users, environmentalists, and other interest groups do not, as a general rule, agree on a common set of objectives and constraints [13].

The core problem consists of designing a transit network capable of transporting a large number of people efficiently and effectively. This problem is highly complex. Standard network design techniques cannot easily be applied to such contexts because the problem is typically of very large scale and involves non-linearities, as well as a multiplicity of criteria. It is more common for planners to generate a set of basic scenarios that are later analyzed, modified and refined. Some authors have proposed various indices to assess the topological configurations of rapid transit systems (see, e.g. Refs. [14-16]) but such indices are more easily used as a way of measuring the quality of proposed or existing networks than as a means of generating good solutions.

Dufourd et al. [17] propose a heuristic approach, based on tabu search, for a simplified version of the problem, where a single transit line is determined such that the total population covered by the alignment is maximized. Their methodology can be equally applied to determine bus lines for this same objective. This approach requires relatively little data and it can easily be used to fine tune a tentative solution. Its main disadvantages are the fact that a real implementation can be time consuming, and the search process can become trapped in a lowquality local optimum. Bruno et al. [18] propose a different heuristic approach for the single alignment problem, which consists of two phases: (1) construction of an initial alignment, and (2) solution improvement. Both papers work on a discretized grid network (square grid of 100 $\mathrm{x} 100)$, where a population $p(x, y)$ is associated with each integer coordinate vertex $(x, y)$. It is assumed that people travel by foot to the closest station using a Manhattan (rectilinear) metric. The total population, covered by a station $s$, is estimated taking a gravitational model of proximity into account. A maximum separation between consecutive stations is imposed, and a minimum separation between any two stations (consecutive or not) must also be added to avoid duplicity when counting passengers in the transit system.

Previous references $[17,18]$ are seminal instances within the scientific literature relative to the effective design of rapid transit systems. Both papers use the frequent objective of maximizing the covered population by means the optimal location of stations, taking only into account the population living close to a potential stop (as happened in Ref. [17]) or combining that objective with data on origin-destination demand (as proposed in Ref. [18]). For a survey on rapid transit network design, see Ref. [19]. As can be seen, the design of rapid transit lines in urban contexts (metro, tram, BRT), by using as main objective the maximization of the functional diversity of the districts that are crossed by the infrastructure, is a problem that has not been yet treated in the literature from a purely scientific perspective.

This paper addresses the problem of locating a rapid transit line with the objective of maximize the functional diversity of the districts traversed by the alignment. Section 2 is devoted to measure the complexity degree of a city by means of the concept of urban entropy. In Section 3 a mathematical model is formulated in order to determine an alignment which maximizes the global entropy. A computational experience has been implemented in Section 4 in order to test the performance of proposed heuristic. Finally, conclusions are summarized in Section 5.

\section{MEASURING THE URBAN SPRAWL}

The analogy between city and information theory was developed in the 1960's, providing through the concept of urban entropy various formal approaches to generate simulation 
models that explain the interaction between the origins and destinations within the city, caused by the functional heterogeneity of the districts.

Shannon's entropy formula measures the diversity of information in a message [20]. Logically, the greater the diversity of data provided in a message, the greater the difficulty in predicting the exact meaning of the transmission. Margalef [21] used a mathematical expression of the entropy to calculate the diversity of species in an ecosystem. He assimilated the different species to symbols of a message, so that the quantity of information of that message is equal to the diversity of species. Note that, if we assume that the different existing species are adapted to the environment, the measure of the diversity gives us also an idea of the degree of the organization of the ecosystem. Later, this methodology has been used by other authors to assess the organization degree or complexity of the urban fabric. Instead of species, the symbols are activities (trades, facilities, offices, services, etc.). Shannon's entropy is a commonly used and effective technique for monitoring and measuring of urban sprawl [22]. For this purpose, Shannon's formula measures the diversity of activities existing in the $i$-th zone and also the global entropy of the city for a given splitting into urban districts. Formally,

where

$$
H_{i}=-\sum_{k=1}^{m} p_{i k} \log _{2}\left(p_{i k}\right) ; H=\sum_{i=1}^{n} H_{i}
$$

- $H_{i}$ is the entropy of the $i$-th zone (or urban district).

- $p_{i k}$ is the probability or proportion of the $k$-th variable (activity) in the $i$-th district.

- $m$ is the total number of zone variables that have been analyzed.

- $H$ is the whole entropy of the city.

- $n$ is the total number of districts considered.

As is emphasized in Ref. [23], the city can be understood as a complex system where there is a continuous exchange of information in the form of flows of people, goods, situations, data, etc. (i.e. species, characteristic variables). Note that the organization of such a system is all the more complex the greater the number of species, and therefore diversity can be understood as an organization measure. In that sense, the greater the value of the entropy, the greater the degree of the system organization. In addition, discontinuities in the urban fabric of the territory represent obstacles to the flow of information. As a result of the increase of accessibility, the interconnection between two zones through a rapid transit system will lead to, in the medium and long terms, the territorial cohesion between both and the effective compensation of its characteristic variables.

As a real application of his thesis, Sierra [24] elaborates a zonal division of the city of Glasgow based on a collection of data concerning to the uses of the buildings, number of floors and diversity of commercial establishments. Moreover, Sierra [24] emphasizes the influence that some zones, provided with high levels of urban entropy, can induce over their adjacent ones. For instance, a district of high complexity transfers such characteristic to the adjacent cells, through connecting streets as influence corridors. The lowest the travel time between adjacent cells, the highest the degree of complexity is transferred.

Bascuñán et al. [25] analyzed the urban areas of La Serena-Coquimbo (Chile) according to the number of services covered at walking distances less than 10 minutes. Empirically, they show in that paper that the higher the entropy level of the zone, the lower the energy consumption and, from an urban perspective, propose the formation of small poly-centers of 
multi-services (more than three different services) that cover, with the help of a rapid transit system, an area of urban population of approximately 78 ha.

\section{ASSESSING THE LOCATION OF A TRANSIT LINE}

The most widely-used decision criteria in transportation network design are related to the maximisation of the population covered by the lines [26]. For instance, the methodology applied to determine the most effective metro lines in terms of trip coverage in the metropolitan area of Seville, illustrated in Figure 1, was based on maximizing the capture of origin-destination traffic. Once the metropolitan area had been divided into macro zones, the generated/attracted trips from/to each zone to/from its adjacent ones were counted. The width of each arrow in Figure 1 is proportional to the number of trips. Line 1 of the Seville Metro, the only one currently built, was the most efficient for the criterion of covering travel demand and was consequently designed to connect zones A. Aljarafe Sur with A.M. Sur.

Since rapid transit systems are designed for the long term, other criteria can be applied to determine alignments which serve as the basis for the construction of public transit infrastructures. Among them, to maximize the global entropy of the metropolitan system, assuming, as was previously pointed out, that the inter-connection of two zones produces homogeneity of their urban characteristics in the medium and long term.

The following properties are necessary to support the performance of the design algorithm proposed to construct a rapid transit line whose objective is the maximization of the global entropy of the urban districts system.

\section{Property 1.}

Let be the function $h(x)=-x \log _{2}(x), x \in(0,1]$. For any pair of values $p, q \in(0,1]$, the following inequality holds: $h(p)+h(q) \leq 2 h\left(\frac{p+q}{2}\right)$.

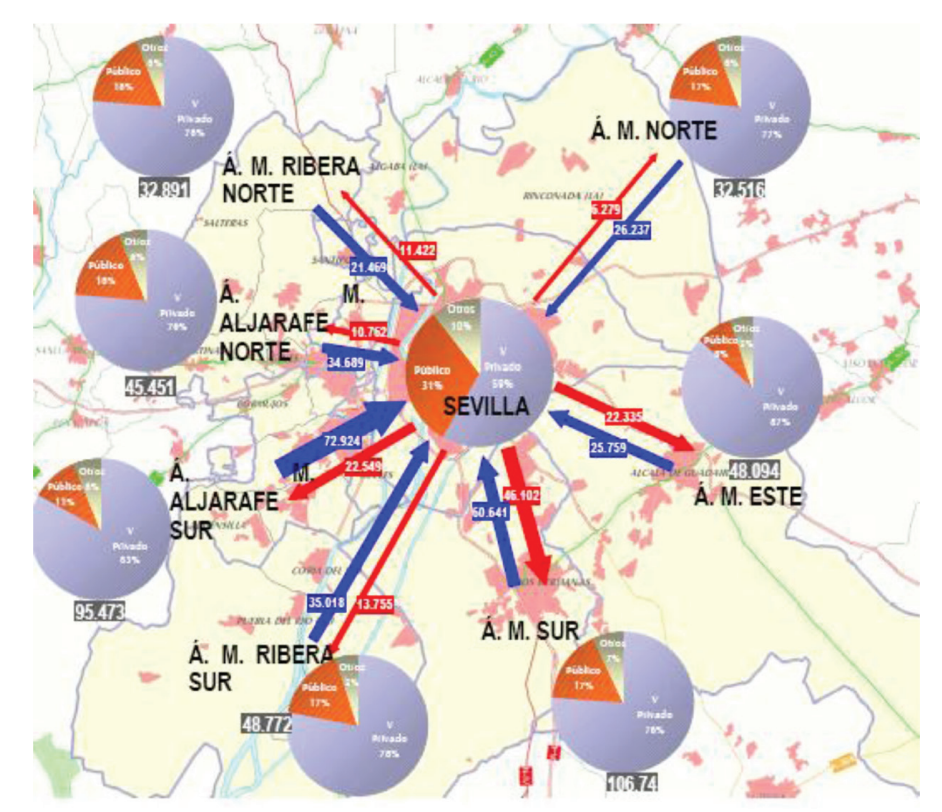

Figure 1: Generated and attracted trips to/from zones in the metropolitan area of Seville. 
Property 2.
Let be the entropic function $H(\vec{x})=-\sum_{k=1}^{m} x_{k} \log _{2}\left(x_{k}\right), x_{k} \in(0,1], \forall k=1, \ldots, m$. For any pair of vectors $\vec{p}=\left(p_{1}, \ldots, p_{m}\right), \vec{q}=\left(q_{1}, \ldots, q_{m}\right) ; p_{k}, q_{k} \in(0,1], \quad$ algebraic expression $2 H\left(\frac{\vec{p}+\vec{q}}{2}\right)-H(\vec{p})-H(\vec{q})$ reaches its minimum value when both vectors $\vec{p}, \vec{q}$ coincide; i.e. for $p_{k}=q_{k}, \forall k=1, \ldots, m$.

The problem of determining an alignment so that global entropy is maximised can be formulated as follows: Given an urban system composed of $n$ districts, where a set of centroids (candidate stations) $S=\left\{s_{1}, \ldots s_{j} \ldots, s_{n}\right\},|S|=n$, represent the different urban areas with their specific characteristics (an unique centroid per zone) collected in the matrix $P=\left\{p_{j k}\right\}, j=1, \ldots, n ; k=1, \ldots, m$, determine a subset $L$ of $S$ and an alignment $\operatorname{Align}(L)$ on points of $L$ such that global entropy is maximized without exceeding a constraint of maximum length (LMAX) for Align(L). Formally,

$$
\operatorname{Max}_{L C S} H(L ; S)=-|L| \sum_{k=1}^{m} r_{k} \log _{2}\left(r_{k}\right)-\sum_{j \in S \backslash L} \sum_{k=1}^{m} p_{j k} \log _{2}\left(p_{j k}\right)
$$

s.t.

$$
\begin{gathered}
r_{k}=\frac{1}{|L|} \sum_{j \in L} p_{j k} ; k=1, \ldots, m \\
\text { Length }[\operatorname{Align}(L)] \leq L M A X .
\end{gathered}
$$

Hence, our measure of effectiveness, derived from having selected a set of nodes to build line $L$, is obtained by additively cumulating the partial entropies of each zone, taking into account that the characteristics of all the zones selected to form the alignment have been recalculated in order to force a common coincidence with their mean values. The objective function to be maximized, formulated by means of expression (2), describes the overall entropy of the urban district system, once a subset of them has been selected for the construction of the transit alignment. The constraint set (3) states that the characteristics of the selected districts must match their mean values. Constraint (4) ensures that the alignment length does not exceed the budget.

On the other hand, the common constraint (see, e.g. Ref. [18]) consisting of imposing that distance between two consecutive stations must lie within an interval $\left[L_{\min }, L_{\max }\right]$, typically [0.5 km, $2 \mathrm{~km}]$, can be easily included in our model, by considering that each district has only one associated centroid and the district size is enough large so that distance between neighbouring centroids lies within the typical variation range.

This problem of combinatorial nature can be assumed as a variant of the Multiple Knapsack Problem (MKP) [27]. The 0-1 Knapsack Problem is the problem of choosing a subset of items such that the corresponding profit sum is maximized, without the weight sum to exceed a prefixed capacity. The Multiple Knapsack Problem is a generalisation of the standard 0-1 Knapsack Problem, where instead of considering only one knapsack, one tries to fill several knapsacks of different capacities. The MKP problem is strongly NP-complete and the need for algorithms that give a good heuristic solution is justified by the computational complexity of this problem. A model similar to the one previously proposed has been investigated in Ref. [28], whereby heuristics were designed in a computationally feasible way and 
consistent with the approach. Tests carried out on randomly generated data have shown that a simple greedy extension heuristic yields the best results if the inter-station spacing is sufficiently large. Otherwise, an insertion heuristic followed by a post-optimization phase is the tool required to obtain the most efficient design.

Taking these precedents into account, we propose heuristic GINA (Greedy Insertion of Nodes along an Alignment) for solving our optimization model for determining the most effective metro line in terms of urban entropy.

\section{Heuristic GINA}

1. Read locations of centroids $\left\{s_{1}, \ldots s_{j} \ldots, s_{n}\right\}$ and their characteristics $\left\{p_{j 1}, \ldots p_{j m}\right\}, \forall j=$ $1, \ldots, n$.

2. Find the pair of centroids $(u, v)$ which generates the most effective link. (In the case of a tie, consider the shortest edge).

3. Let $\operatorname{Align}(L)=\{u, v\}$.

4. While length of alignment Align $(L)$ is less than boundary LMAX:

4.1 Find the node $w^{*}$, not yet included in $\operatorname{Align}(L)$, where the highest effectiveness is reached. (In the case of a tie, consider the option which generates a minor increase in length).

4.2 Determine the position $\operatorname{pos}\left(w^{*}\right)$ where node $w^{*}$ should be inserted along the node sequence $\operatorname{Align}(L)$ to produce the smallest increase in its length.

4.3 Insert node $w^{*}$ at position $\operatorname{pos}\left(w^{*}\right)$ along the sequence $\operatorname{Align}(L)$. Rename the new resulting alignment, once added node $w^{*}$, as $\operatorname{Align}(L)$.

\section{COMPUTATIONAL EXPERIENCE}

A computational experience has been carried out on a district system (composed of 47 zones associated to the metropolitan area of Seville) in order to test the performance of heuristic GINA. The experiment consists of designing a rapid transit line connecting nine city zones by means an alignment passing through them, using two different criteria: maximizing the population covered (assuming that the inhabitants are covered by the transport service if they live in an area whose centroid is included in the transit corridor) and maximize urban entropy (the areas connected through the corridor share the same level for the attributes considered in the analysis of urban diversity). First objective was already applied in Ref. [18] to locate one alignment on data from the city of Milan. Second objective, unpublished at this time, is focused on increasing the diversity of the interconnected districts.

Based on data corresponding to the Statistical Yearbook 2015 of Seville (available at http:// www.sevilla.org/ayuntamiento/competencias-areas/area-de-h/anuario_2015), values of seven characteristics (population, medical services, job positions, intermodality, study centres, commercial centres and tourist attractions) have been estimated and normalized for the 47 zones under consideration. Such attributes were already identified in Ref. [13] as the main mobility generators. Hence, seven different intensity maps can be obtained when each attribute is separately considered. Between them, as instances, in Figure 2 normalized population densities are shown on the district map; on the other hand, Figure 3 shows the distribution of densities of tourist attractions.

Applying the GINA algorithm on this Seville map, the alignment is generated in an iterative way. In the first step, the zone with the highest entropy level (La Calzada, the 24th zone) is connected to the area with the lowest level (Isla Mágica, the 41th zone). Next, different nodes are incorporated in sequence to form the alignment of 9 nodes that produces a greater 


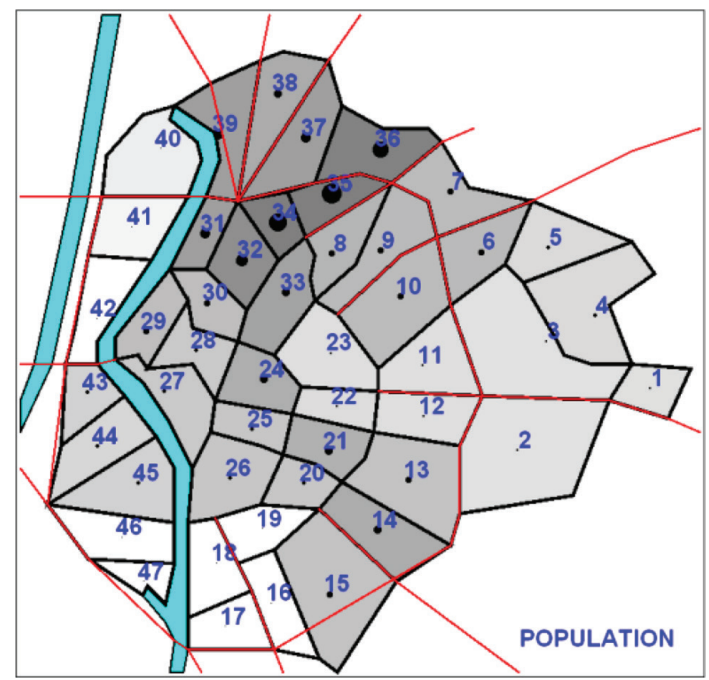

Figure 2: District system (47 zones) associated to Seville. Map of normalized densities of population (Source: Laporte et al., 2002.).

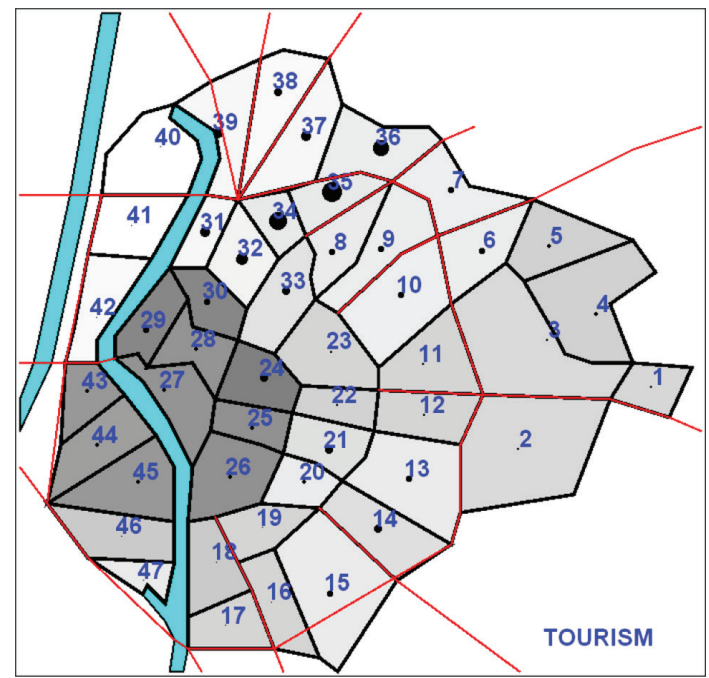

Figure 3: District system (47 zones) associated to Seville. Map of normalized densities of tourist attractions.

entropy level for the global system: $\{46,18,24,30,29,41,40,34,35\}$ (the length of that line, shown in Figure 4, is 13889 meters). Initially, the entropy had the value 35.8011; at the end of the process, the value of the entropy amounts to 36.8560 (2.94\% higher).

On the other hand, by using the maximization of the covered population, as a criterion of node incorporation in the alignment, we obtain an optimal corridor composed of nodes $\{38,39,31,32,33,34,35,37,36\}$ (the line length shown in Figure 5 is 8960 meters). This alignment has an entropy value of $36.0856,2.13 \%$ lower than previously achieved. 


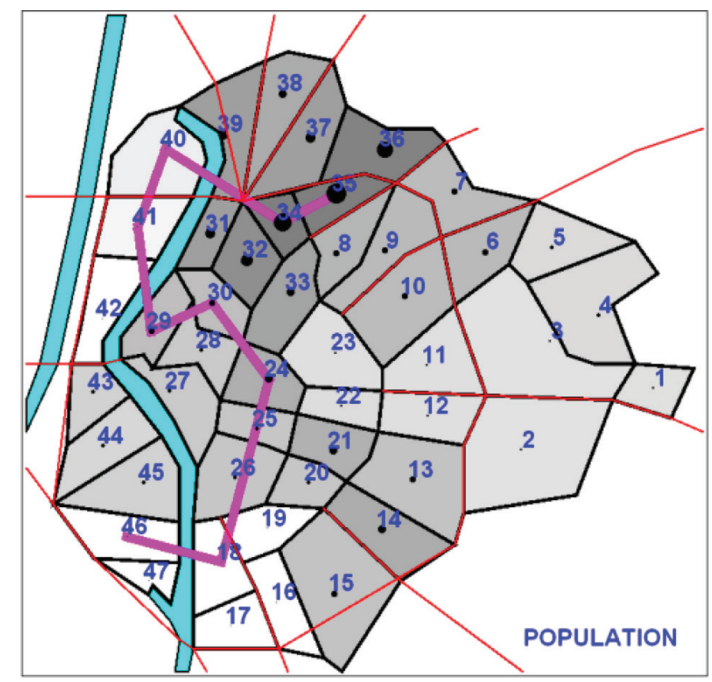

Figure 4: 9-station line obtained for the criterion of maximizing the global urban entropy.

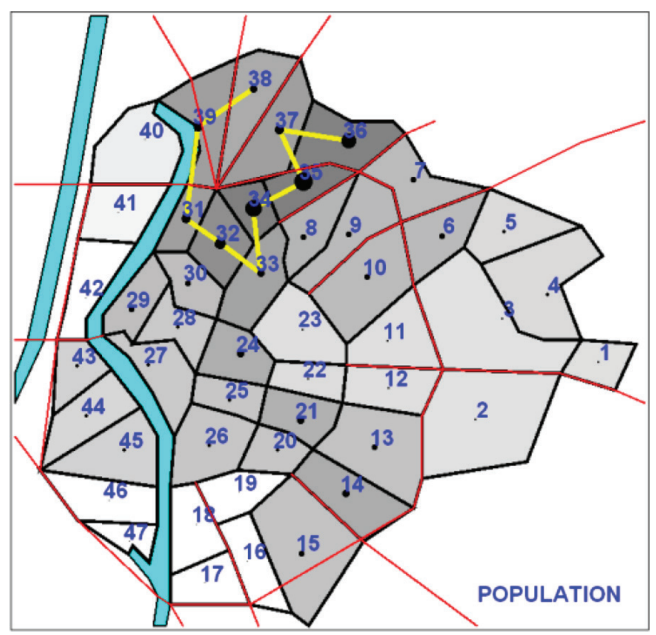

Figure 5: 9-station line obtained for the criterion of maximizing the population covered.

First alignment, obtained by applying a criterion of maximizing the global urban entropy, presents two desired advantages: from an urban planning perspective, the improvement of the territorial cohesion within the district system of the city, and, from the point of view of sustainability, the reduction of the forced mobility of the inhabitants caused by the lack of opportunities of the districts where they live.

\section{CONCLUSIONS}

A methodology for the design of a rapid transit alignment, that increases the urban entropy of a district system, has been proposed in this article. A greedy algorithm has been formulated to solve the proposed mathematical programming model, identified as a version of the 
well-known problem of Multiple Knapsack Problem. To evaluate the performance of the proposed methodology, a computational experience has been carried out on an urban system composed of 47 districts with data from the metropolitan area of Seville (Spain). The evaluation of the generated transit line shows that the methodology meets the objective of efficiently designing an alignment that improves the functional diversity of the areas where it crosses.

\section{ACKNOWLEDGEMENTS}

This research has been partially supported by the Spanish Ministry of Economy and Competitiveness through grant MTM2015-67706-P (MINECO/FEDER, UE). This support is gratefully acknowledged.

\section{REFERENCES}

[1] Bhatta, B., Analysis of urban growth and sprawl from remote sensing data. Advances in Geographic Information Science, pp. 17-36, 2000. https://doi.org/10.1007/978-3-642-05299-6_2

[2] Zhang, T., Community features and urban sprawl: the case of the Chicago metropolitan region. Land Use Policy, 18(3), pp. 221-232, 2001. https://doi.org/10.1016/s0264-8377(01)00018-7

[3] Mosammam, H.M., Nia, J.T., Khani, H., Teymouri, A. \& Kazemi, M., Monitoring land use change and measuring urban sprawl based on its spatial forms. The Egyptian Journal of Remote Sensing and Space Science, 20(1), pp. 103-116, 2016. http://dx.doi.org/10.1016/j.ejrs.2016.08.002

[4] Rodrigue, J.-P., The geography of transport systems, Routledge: New York, 2017. Available at: https://people.hofstra.edu/geotrans/eng/ch6en/conc6en/ch6c4en.html. ISBN 978-1138669574. (Accessed 5 April 2017).

[5] Salat, S. \& Bourdic, L., Urban complexity, scale hierarchy, energy efficiency and economic value creation. In WIT Transactions on Ecology and the Environment, Vol. 155, The Sustainable City VII: Urban Regeneration and Sustainability, Vol. 1 (Eds. M. Pacetti, G. Passerini, C.A. Brebbia, G. Latini) pp. 97-107, 2012.

[6] Burton, E., The compact city: Just or just compact? A preliminary analysis. Urban Studies, 37(11), pp. 1969-2001, 2000. https://doi.org/10.1080/00420980050162184

[7] CEC, Sustainable Urban Development in the European Union: A Framework for Action, Communication from the Commission COM (1998) 605 final, 28.10.98, Commission of the European Communities: Brussels, 1998.

[8] CEC, A new partnership for cohesion. Convergence, Competitiveness, Cooperation, Third Report on Economic and Social Cohesion, Commission of the European Communities: Brussels, 2004.

[9] Vickerman, R., Spiekermann, K. \& Wegener, M., Accessibility and economic development in Europe. Regional Studies, 33(1), pp. 1-15, 1999. https://doi.org/10.1080/00343409950118878

[10] Martín, J.C., Gutiérrez, J. \& Román, C., Data Envelopment Analysis (DEA) index to measure the accessibility impacts of new infrastructure investments: the case of the high-speed train corridor Madrid-Barcelona-French border. Regional Studies, 38(6), pp. 697-712, 2004. https://doi.org/10.1080/003434042000240987 
[11] López, E., Gutiérrez, J. \& Gómez, G., Measuring regional cohesion effects of largescale transport infrastructure investments: an accessibility approach. European Planning Studies, 16(2), pp. 277-301, 2008. https://doi.org/10.1080/09654310701814629

[12] López, E. \& Monzón, A., Integration of sustainability issues in strategic transportation planning: a multi-criteria model for the assessment of transport infrastructure plans. Computer-Aided Civil and Infrastructure Engineering, 25, pp. 440-451, 2010. https://doi.org/10.1111/j.1467-8667.2010.00652.x

[13] Gendreau, M., Laporte, G. \& Mesa, J. A., Locating rapid transit lines. Journal of Advanced Transportation, 29, pp. 145-162, 1995. https://doi.org/10.1002/atr.5670290202

[14] Musso, A. \& Vuchic, V.R., Characteristic of metro networks and methodology for their evaluation. Transportation Research Record, 1162, pp. 22-33, 1988.

[15] Laporte, G., Mesa, J.A. \& Ortega, F., Assessing topological configurations for rapid transit networks. Studies in Locational Analysis, 7, pp. 105-121, 1994.

[16] Laporte, G., Mesa, J.A. \& Ortega, F., Assessing the efficiency of rapid transit configurations. TOP, 5, pp. 95-104, 1997. https://doi.org/10.1007/bf02568532

[17] Dufourd, H., Gendreau, M. \& Laporte, G., Locating a transit line using tabu search. Location Science, 4, pp. 1-19, 1996. https://doi.org/10.1016/s0966-8349(96)00008-3

[18] Bruno, G., Gendreau, M. \& Laporte, G., A heuristic for the location of a rapid transit line. Computers \& Operations Research, 29, pp. 1-12, 2002. https://doi.org/10.1016/s0305-0548(00)00051-4

[19] Laporte, G., Mesa, J.A., Ortega, F.A. \& Perea, F., Planning rapid transit networks. Socio-Economic Planning Sciences, 45, pp. 95-104, 2011. https://doi.org/10.1016/j.seps.2011.02.001

[20] Shannon, C.E., A mathematical theory of communication. Bell System Technical Journal, 27, pp. 379-423, 1948. https://doi.org/10.1002/j.1538-7305.1948.tb01338.x

[21] Margalef, R., Teoría de los sistemas ecológicos, Publicacions de la Universitat de Barcelona. Barcelona, 1991.

[22] Yeh, A.G.O. \& Xia, L., Measurement and monitoring of urban sprawl in a rapidly growing region using entropy. Photogrammetric Engineering \& Remote Sensing, 67(1), pp. 83-90, 2001.

[23] Coward, A. \& Salingaros, N., The information architecture of cities. Journal of Information Science, 30(2), pp. 107-118, 2004. https://doi.org/10.1177/0165551504041682

[24] Sierra, M., Towards an ecology of the form. Game theory and urban sustainability in the information age, Doctoral dissertation, Universidad de Sevilla, 2009.

[25] Bascuñán-Walker, F., Bordones-Gana, D. \& Reyes-Fernández, J., Efectos de la entropía urbana en el coste energético del trasporte. Urbano, 14(23), pp. 20-27, 2011. (Universidad del Bío Bío, Concepción, Chile.)

[26] Mesa, J.A. \& Ortega, F.A., Park-and-ride station catchment areas in metropolitan rapid transit systems, M. Pursula and J. Niittymäki (eds.), Mathematical Methods on Optimization in Transportation Systems, (pp. 81-93) Kluwer Academic Publishers, Dordrecht, Netherlands, 2001. 
[27] Pisinger, D. \& Toth, P., Knapsack problems, in: D-Z. Du \& P. Pardalos (Eds.), Handbook of Combinatorial Optimization, Vol. 1, Kluwer Academic Publishers, Dordrecht, pp. 299-428, 1998.

[28] Laporte, G., Mesa, J.A. \& Ortega, F., Determinación del Alineamiento de Máxima Cobertura de Viaje en el Diseño de Sistemas Urbanos de Transporte (in Spanish). Proceedings of the V Congreso de Ingeniería del Transporte, pp. 2179-2188, 2002, Santander, Spain. 